\title{
Study on the Simulation of Hot Event's Propagation Based on Ant Colony Algorithm in Social Networks
}

\author{
D.C. Qu, H. Xie, H.X Lian, J. Bai \\ School of Computer Science \& Technology \\ Beijing Institute of Technology \\ Beijing, China
}

\author{
S.L. Guo \\ School of Automation \\ Beijing Institute of Technology \\ Beijing, China
}

\begin{abstract}
Given that there is not much research on the propagation mechanism of hot event in the social networks environment at the macro level at present, this paper proposes an optimizing model of propagation to maximize the influence of hot event in networks in a short time. Combining the three characteristics of basic ant colony algorithm: distributive, selforganizing and positive feedback, a hot event's propagation model based on improved ant colony algorithm is built. The optimization goal of this model is to maximize the number of users who concern about a networking event in a short time. Through experiment, the optimizing propagation of hot events is simulated, different periods of evolution are shown, and the effectiveness of the model is validated.
\end{abstract}

Keywords-social networks; networking hot event; simulation of propagation; ant colony algorithm

\section{INTRODUCTION}

The study of the evolvement of hot event's propagation is one of the hottest research fields. Related interdisciplinary studies include: "the psychology of conformity" Sznajd model in social psychology, which is used to implement the growth and preferential attachment of the scale-free networks, so as to promote the formation of a propagation path [1]; the dynamical model of continuous view-model is proposed by Deffaunt et al. Different from the dualistic-view and discreteview models, Deffaunt proposes that a viewpoint can be any value in the [0,1] continuous interval [2]; The SIS and SIR models based on ordinary differential equation figure out that various diseases have critical behaviors and the feature of thresholds, but they both make the assumption of well-mixed, that individuals have the same probability of contact, the influence of networking topological structure on the propagation performance is ignored [3]; when American social psychologist B.Latane does research on the public opinion propagation, he proposes a social impact model, considering the factors of individuals' social influences, such as the spatial distance between individuals, closeness of relationship, social status, etc. And the public opinion dynamical model of social impact is built [4]. However, on social networking platforms, there are comparatively few studies on the hot event propagation at macro level.

In this paper, the propagation of hot event in social networking environment is defined as follows [5]: ordinary social networking users send messages(such as posts, instant messages, tweets, weibo messages and so on), which cover the hot social issues and sensitive topics. Due to the scalefree distributive and small-world characteristics of social networking topological structure and plenty of users' interaction, these messages are widely read, replied and reposted in a short time, and then these messages become the headlines and hot topics of the social networking platforms.

In terms of behaviors and characteristics, there are similarities between the ant's feeding process and hot event's propagation process in networks. Firstly, the individuals are independently distributed. In the process of hot event's propagation in networking structure, every user node can be regarded as independent solution to "solve the hot event problem in networks". The distribution of hot events has no center and is scale-free, any user can be the initiator of a hot event, which is in accordance with the independent distribution characteristic of ant colony algorithm. Secondly, the individuals are Self-organized. In the process of propagation of hot event in networks, users interact with each other, the information is constantly reposted and spread, the whole process is self-organized by users. While the information exchanges between ants are also spontaneous actions. Thirdly, there is positive feedback feature in both the process. From a psychological perspective, the networking users' psychology of conformity is an important driving force for the formation and propagation of hot event in networks, the pheromone interaction of ant colony algorithm is also a typical application of positive feedback mechanism [6].

II. STUDY OF THE SOCIAL NETWORKING ENVIRONMENT AND THE MACRO-FEATURE OF HOT EVENT'S PROPAGATION

With the rapid development of social networks, more and more studies focus on the propagation evolution of hot event in networks. Different from ordinary networks, such as random graph, social networks are in accordance with complex networks [7]. One of the important features of complex networks is: small-world effect proposed by Watts and Strogatz in 1998 [8]. Leskovec and Horvitz showed that the distance of any two people is 6.6 by using a huge amount of real time information networks including 18 million users in 2008 [9]. Because of the complex-networking features and the instant interactions, event propagation in social networks are more likely to present emerging phenomenon.

To study the overall evolving properties of hot event's propagation, the primary task is to describe the macro-states on the whole. The macro-description is often measured by the result of propagation. Generally the transformation of hot event and cold event is the macro phenomenon, and how the 
information transfers between "hot" and "cold" in the process is formed through micro interactions. A typical model is built by $\mathrm{Li}$ Zhihong to describe the overall emergence process which is separated into five periods: the precursor period, the emerging period, the extensive period, the relief period, and the termination period [10].

To study the propagation of hot event, especially the emerging phenomenon, the termination period is ignored in this paper. Thus the propagation of hot event in social networks are separated into the four periods.

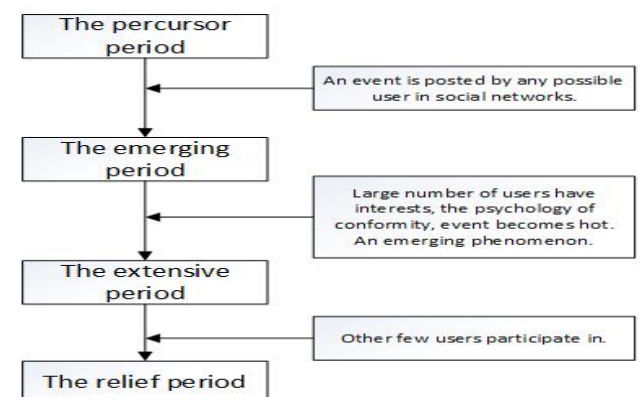

FIGURE I. FOUR PERIODS SEPARATED FOR THE MACROPROPAGATION OF HOT EVENT.

\section{The OPTIMIZING PROPAGATION MODEL OF HOT EVENT}

Suppose there are $M$ nodes in a social networking environment, where $M$ is the number of users in the networks. $\mathrm{X}$ is presented as one event, $\sigma(\mathrm{X})$ denotes the number of users concerning $X$, then $\sigma(X) \leq M$. The problem of maximizing the propagation is to maximize $\sigma(\mathrm{X})$ in a short time:

$$
\max _{i \in M} \sigma(\mathrm{X}) . \sigma(\mathrm{X}) \leq N, \operatorname{Min}(t)
$$

The user number of social networking can be unlimited, then the optimization of the propagation is a NP problem, which has no polynomial solution. Ant colony algorithm is one kind of combinatorial optimization algorithms for solving the combinatorial optimization NP problem [11].

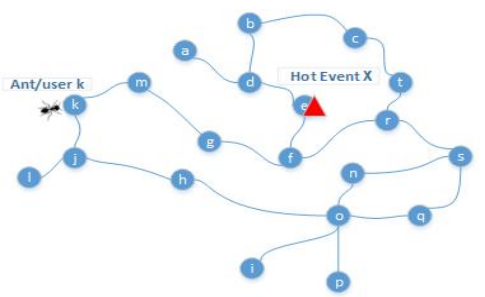

FIGURE II. A SIMPLE TOPOLOGICAL STRUCTURE OF A SOCIAL NETWORK.

The application of ant colony algorithm to the simulation of hot event $\mathrm{X}$ propagation can be described as: event $\mathrm{X}$ is randomly proposed by one of the nodes, and another user node(ant) $\mathrm{k}$ finds the node with event $\mathrm{X}$ through links of friends. $\mathrm{k}$ leaves pheromone to attract other users(ants) to find event $\mathrm{X}$ in one shortest time that formatting a short path with the smallest number of nodes. With positive feedback of pheromone, other ants find event $X$ in a short time. When the number of users find event $X$ is large, there is an emerging phenomenon, and event $\mathrm{X}$ becomes hot. Learn from the ant colony algorithm for TSP problem [12], the symbols and their meanings in the ant colony algorithm for optimizing model are given as follows:

$$
\boldsymbol{u}_{\boldsymbol{i}} \text {-User i; }
$$

$\boldsymbol{d}_{\boldsymbol{i} \boldsymbol{j}}$-The number of user nodes on the path from user $\mathrm{i}$ to user $\mathrm{j}$;

m - The number of ants;

$\boldsymbol{\tau}_{i j}(\mathbf{t})$-At time $\mathrm{t}$, the pheromone leaved on the path $\boldsymbol{u}_{\boldsymbol{i}}$ $\boldsymbol{u}_{j}$, at beginning, the pheromone on every path is the same;

$\boldsymbol{\tau}_{\boldsymbol{i} \boldsymbol{j}}^{\boldsymbol{k}}(\boldsymbol{t})$-At time $\mathrm{t}$, the pheromone ant $\mathrm{k}$ leaves on the path $\boldsymbol{u}_{\boldsymbol{i}}-\boldsymbol{u}_{\boldsymbol{j}}$;

$\boldsymbol{\eta}_{i j}(\mathbf{t})$-At time $\mathrm{t}$, the heuristic information on the path $\boldsymbol{u}_{\boldsymbol{i}}-\boldsymbol{u}_{\boldsymbol{j}}$, generally is $1 / d_{i j}$, denotes that the path with smaller number of users is preferred.

$\boldsymbol{N}_{\boldsymbol{i}}^{\boldsymbol{k}}(\mathbf{t})$ - At time t, at node i, the collection of nodes that ant $\mathrm{k}$ can visit next;

$\boldsymbol{p}_{\boldsymbol{i} \boldsymbol{j}}^{\boldsymbol{k}}(\boldsymbol{t})$ - the probability that ant $\mathrm{k}$ transfer from user node $\boldsymbol{u}_{\boldsymbol{i}}$ to $\boldsymbol{u}_{j}$.

\section{A. The Model Design}

1) Initialization: Randomly choose one node as the hot event source, and randomly distribute $\mathbf{m}$ ants on the nodes. Every path is initialized with the same pheromone [13].

2) The rules of hot events propagation based on ant colony algorithm: At time $\mathbf{t}$, ant $\mathbf{k}$ arrives user node $\mathbf{u}_{\mathbf{i}}$, ant $\mathbf{k}$ chooses the next node $\mathbf{u}_{\mathbf{j}}$ with the largest $\mathbf{p}_{\mathbf{i j}}^{\mathbf{k}}(\mathbf{t})$ according to the following eqn (1) [14], and there must have links between node $\mathbf{u}_{\mathbf{i}}$ and node $\mathbf{u}_{\mathbf{j}}$ :

$$
= \begin{cases}\frac{\tau_{i j}^{\alpha}(t) \eta_{i j}^{\beta}(t)}{\sum_{r \in N_{r}^{k}(\mathrm{u})} \tau_{i r}^{\alpha}(t) \eta_{i r}^{\beta}(t)}, & j \in N_{i j}^{k}(\mathrm{t}) \\ 0, & j \notin N_{i j}^{k}(\mathrm{t})\end{cases}
$$

$\alpha$ is the importance of pheromone on the path, $\beta$ is the importance of the heuristic information. Ants will not repeat the selection of the same user node in social networking structure.

3) The updating rules of Pheromone with interest and participation: Based on the basic ant colony algorithm, the updating rules of pheromone are improved with interest and participation in this paper. In the propagation of hot event, the users with great interests choose to concern the event, the participation of users also have great impact on the propagation. The interest value $\boldsymbol{\theta}$ and the participation value $\boldsymbol{\varepsilon}$ are used to improve the pheromone on the path the ants have passed by. After the ants finish all the traversal of the nodes they can travel to, the pheromone is updated.

In this paper, the updating rule for the paths that ants haven't passed by is as follows, eqn (2):

$$
\tau_{i j}(t+1)=(1-\rho) * \tau_{i j}(t), \quad \rho \in
$$


The updating rule for the path that ants have passed by is as follows, eqn (3):

$$
\begin{gathered}
\tau_{i j}(t+1)=(1-\rho) * \tau_{i j}(t)+(1+\theta \varepsilon) \Delta \tau_{i j}, \rho \in \\
\quad(0,1) \\
\Delta \tau_{i j}=\sum_{k=1}^{n} \Delta \tau_{i j}^{k} \\
\Delta \tau_{i j}^{k}=\frac{Q}{L_{k}}
\end{gathered}
$$

$\mathrm{Q}$ is a constant value, $\mathrm{L}_{\mathrm{k}}$ denotes the number of user nodes that ant $\mathrm{k}$ has traveled to. $\rho$ denotes the degree of volatilization of pheromone, and $0<\rho<1 . \Delta \tau_{i j}$ denotes the global updating factor of pheromone. $\theta$ is the interest of users in event $\mathrm{X} . \varepsilon=\mathrm{C}(\mathrm{t}) / \mathrm{D}(\mathrm{t})$ is the participation of users, $C(t)$ is the number of users at time $t$ participate in event $\mathrm{X}$, and $\mathrm{D}(\mathrm{t})$ is the total number of users.

\section{EXPERIMENTAL SIMULATION AND ANALYSIS}

\section{A. The Analysis of Parameter Values}

The existing research results show that the values of parameters play an important role in the performance of ant colony algorithm. In this experiment, the method of setting values for algorithms provided by Ye Zhiwei et al. is used [15]. Dynamically adjust the parameter values in the selection of path. In the first fourth of expected loops, set the values small $\alpha=0.5, \beta=1, \rho=0.3$. With the increase of loops, adjust the values $\alpha=1, \beta=5, \rho=0.1$. Set $\theta=0.8$ as users have much interests.

\section{B. Experimental Simulation}

In order to relieve the impact of uncertainty and randomness, average statistical method is used for the time of 20 number of users finding the hot event source. After several tests, we find that the total runtime is about 5 mins. In the process of simulation, the number of ants finding the event is recorded every 15 seconds. Five times average result is shown in Table 1.

TABLE I. THE RESULT OF AVERAGE STATISTICAL METHOD.

\begin{tabular}{|ll|ll|ll|ll|}
\hline $\begin{array}{c}\text { Time( } \\
\text { s) }\end{array}$ & Ants & $\begin{array}{l}\text { Time( } \\
\text { s) }\end{array}$ & $\begin{array}{l}\text { Ant } \\
\text { s }\end{array}$ & $\begin{array}{l}\text { Time(s } \\
\text { ) }\end{array}$ & Ants & $\begin{array}{l}\text { Time(s } \\
\text { ) }\end{array}$ & Ants \\
\hline 15 & 0 & 90 & 3.4 & 165 & 12.4 & 240 & 16.6 \\
\hline 30 & 0.6 & 105 & 4.8 & 180 & 13.6 & 255 & 17.2 \\
\hline 45 & 1.2 & 120 & 6.4 & 195 & 14.4 & 270 & 18.2 \\
\hline 60 & 1.8 & 135 & 9.4 & 210 & 15 & 285 & 19.2 \\
\hline 75 & 2.6 & 150 & 11.4 & 225 & 15.8 & 300 & 19.6 \\
\hline
\end{tabular}

The propagation trend is as Figure 3. The result shows the four periods of propagation.

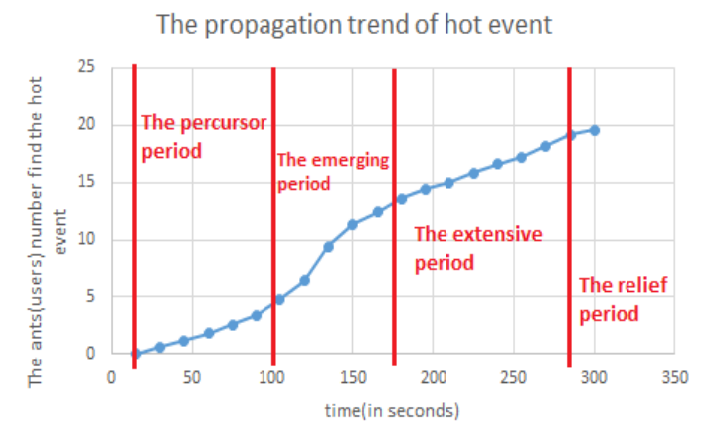

FIGURE III. THE PROPAGATION TREND.

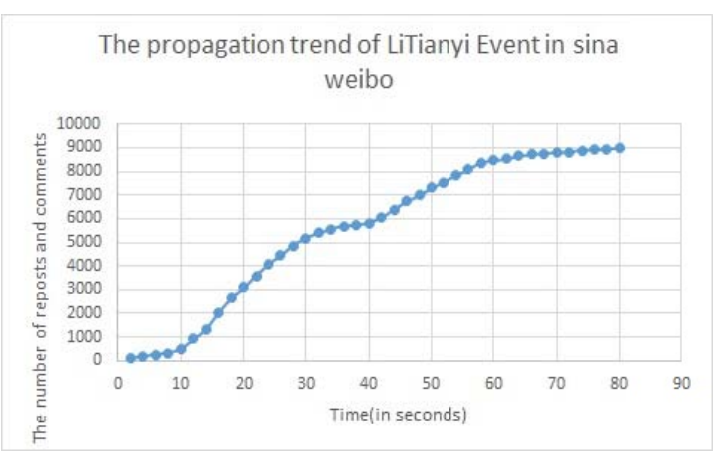

FIGURE IV. THE PROPAGATION TREND OF LI TIANYI EVENT.

\section{The Analysis with Real-World Dataset from Sina weibo Social Networks}

This paper chooses the real world dataset of the famous "Li Tianyi event" in 2013 from sina weibo. Record the number of comments and reposts every 2 hour, the statistical propagation trend result is as Figure 4.

\section{CONCLUSION AND FUTURE WORK}

The real-world dataset shows that the propagation of hot event is in accordance with the simulation, thus the optimizing propagation model simulates the propagation of hot event successfully. More researches on the maximizing of hot event propagation at micro-level will be done in the future.

\section{ACKNOWLEDGEMENTS}

The study is supported by the Natural Science Foundation of China(NSFC) under Grant No. 61370136, and thanks for help of professors and students in Beijing Engineering Research Centre of Massive Language Information Processing and Cloud Computing Application.

\section{REFERENCES}

[1] Stauffer D, De Oliveira, P. M. C., Persistence of opinion in the Sznajd consensus model: computer simulation. The European Physical Journal B-Condensed Matter, 30(4), pp. 587-592, 2002.

[2] Guillaume Deffuant, David Neau, Frederic Amblard et al., Mixing beliefs among interacting agents. Advances in Complex Systems, 3(4), pp. 87-98, 2000.

[3] Artalejo JR, Lopez-Herrero MJ, The SIS and SIR stochastic epidemic models: a maximum entropy approach. Theoretical Population Biology, 80(4), pp. 256-264, 2011. 
[4] James H.Liu, Bibb Latane, Extremitization of attitudes: does thought and discussion induced polarization cumulate. Basic and Applied Social Psychology, 20(2), pp. 103-110, 1998.

[5] Li Biao, The spatial structure and time structure of the propagation of networking hot issues. People's Daily Publishing Corporation, Beijing, China, pp. 14, 2011.

[6] Marco Dorigo, L.M.Gambardella, Luca Maria Gambardella, Ant colony system: a cooperative learning approach to the traveling salesman problem. IEEE Transactions on Evolutionary Computation, 1(1), pp. 53-66, 1997.

[7] Lei Tang, Huan Liu, Community Detection and Mining in Social Media. China Machine Press, Beijing, China, pp. 1-2, 2013.

[8] Watts Duncan J., Strogatz Steven H., Collective dynamics of "smallworld" networks. Nature, 393(6684), pp. 440-442, 1998.

[9] Jure Leskovec, Eric Horvitz, Planetary-scale views on a large instantmessaging network. Proceeding of the 17th international conference on World Wide Web, pp. 915-924, 2008.

[10] Li Zhihong, He Jile, Wu Pengfei et al., The inherent features of dissemination mode of sudden public crisis and the management strategies. Library and Information Service, 51(10), pp. 90, 2007.

[11] Gao Shang, Zhang Lei, Zhuang Fengting, Zhang Chunxian, Solving traveling salesman problem by ant colony optimization algorithm with association rule. The 3rd International Conference on Natural Computation, pp. 693-698, 2007.

[12] Liu Xiabi, Introduction to Artificial Intelligence-methods and system. National defence of Industry Press, Beijing, China, pp. 257-262, 2008

[13] Jia Gangli, Yang Jiaben, Ant colony algorithm with adaptive adjustment of pheromone. Information and Control, 31(3), pp. 198201, 2002.

[14] Yang Jianfeng, Ant colony algorithm and its application. Zhejiang University, Electrical Engineering, Hangzhou, China, pp. 19-44, 2007.

[15] Ye Zhewei, Zheng Zhaobao, The setting of the parameters $\alpha, \beta, \rho$ of ant colony algorithm-use the TSP problem. Journal of Wuhan University, 29(7), pp. 598-601, 2004.

[16] Qu Dacheng, Xin Wei, Zhang Quan, Guo Shuli, The New Analysis Methods of Hot Events Propagation Based on Interdisciplinary Studies. The 4th national conferences on social computing, Beijing, China, pp. 24-30, 2012. 